\title{
Monte Carlo study of liquid crystal phases of hard and soft spherocylinders
}

A. Cuetos, B. Martínez-Haya, L. F. Rull, and S. Lago

Citation: The Journal of Chemical Physics 117, 2934 (2002);

View online: https://doi.org/10.1063/1.1491872

View Table of Contents: http://aip.scitation.org/toc/jcp/117/6

Published by the American Institute of Physics

\section{Articles you may be interested in}

A re-examination of the phase diagram of hard spherocylinders

The Journal of Chemical Physics 104, 6755 (1996); 10.1063/1.471343

Tracing the phase boundaries of hard spherocylinders

The Journal of Chemical Physics 106, 666 (1997); 10.1063/1.473404

Orientational ordering and phase behaviour of binary mixtures of hard spheres and hard spherocylinders The Journal of Chemical Physics 143, 044906 (2015); 10.1063/1.4923291

A new anisotropic soft-core model for the simulation of liquid crystal mesophases

The Journal of Chemical Physics 128, 044906 (2008); 10.1063/1.2825292

Columnar phases of discotic spherocylinders

The Journal of Chemical Physics 129, 214706 (2008); 10.1063/1.3028539

A novel orientation-dependent potential model for prolate mesogens

The Journal of Chemical Physics 122, 024908 (2004); 10.1063/1.1830429

\section{AIP | the Joumal of Chemical Physics}

PERSPECTIVES 


\title{
Monte Carlo study of liquid crystal phases of hard and soft spherocylinders
}

\author{
A. Cuetos and B. Martínez-Haya ${ }^{\text {a) }}$ \\ Departamento de Ciencias Ambientales, Universidad Pablo de Olavide, 41013 Sevilla, Spain \\ L. F. Rull \\ Departamento de Física Atómica, Molecular y Nuclear, Area de Física Teórica, Universidad de Sevilla, \\ Apdo. 1065, 41080 Sevilla, Spain \\ S. Lago \\ Departamento de Ciencias Ambientales, Universidad Pablo de Olavide, 41013 Sevilla, Spain
}

(Received 2 January 2002; accepted 15 May 2002)

\begin{abstract}
We report on a Monte Carlo study of the liquid crystal phases of two model fluids of linear elongated molecules: (a) hard spherocylinders with an attractive square-well (SWSC) and (b) purely repulsive soft spherocylinders (SRS), in both cases for a length-to-breadth ratio $L^{*}=5$. Monte Carlo simulations in the isothermal-isobaric ensemble have been performed at a reduced temperature $T^{*}=5$ probing thermodynamic states within the isotropic (I), nematic $(\mathrm{N})$, and smectic A ( $\left.\mathrm{Sm} \mathrm{A}\right)$ regions exhibited by each of the models. In addition, the performance of an entropy criterion to allocate liquid crystalline phase boundaries, recently proposed for the isotropic-nematic transition of the hard spherocylinder (HSC) fluid, is successfully tested for the SWSC and the SRS fluids and furthermore extended to the study of the nematic-smectic transition. With respect to the more extensively studied HSC fluid, the introduction of the attractive square well in the SWSC model brings the $\mathrm{I}-\mathrm{N}$ and $\mathrm{N}-\mathrm{Sm} \mathrm{A}$ transitions to higher pressures and densities. Moreover, the soft repulsive core of the SRS fluid induces a similar but quite more significant shift of both of these phase boundaries toward higher densities. This latter effect is apparently in contrast with very recent studies of the SRS fluid at lower temperatures, but this discrepancy can be traced back to the different effective size of the molecular repulsive core at different temperatures. (C) 2002 American Institute of Physics. [DOI: 10.1063/1.1491872]
\end{abstract}

\section{INTRODUCTION}

The current understanding of the behavior of liquid crystalline mesogens relies largely on detailed studies of simple molecular fluid models. Over the past decades, a number of models of varying complexity have been developed with the aim of incorporating the essential features of the real systems, while minimizing the expense of their theoretical and numerical treatment. Such expense is mainly imposed by the intrinsic orientational dependence of the pair interaction and the long-range order characteristic of the liquid crystal phases. An important class of such liquid crystal models encompasses fluids of linear molecules interacting via a hard or soft repulsive core of either ellipsoidal or spherocylindrical symmetry, eventually dressed by attractive interactions of different nature. ${ }^{1-5}$ In particular, the hard spherocylinder fluid (HSC) constitutes one of the most extensively studied models and, in spite of its simplicity and purely repulsive interaction, it has been found to display differentiated isotropic, nematic, smectic, and solid phases. ${ }^{1,3}$

In the present work, we have concentrated on two of the simplest modifications of the HSC model which, perhaps surprisingly, have not deserved as much attention as other elemental liquid crystal models: a fluid of hard spherocylinders with an attractive square-well (SWSC), ${ }^{6-8}$ and a fluid of

\footnotetext{
a) Author to whom correspondence should be addressed. Electronic mail: bmarhay@dex.upo.es
}

soft repulsive spherocylinders (SRS). ${ }^{5,9-11}$ We hence intend to explore the effect of the simple features introduced in these two models with respect to the HSC fluid, namely the presence of an attractive well or the softness of the short range repulsive interaction, on the liquid crystal phase diagram of the fluid. In addition, unlike the HSC fluid, these models allow for the study of systems at finite temperature in a natural way. One further fundamental aspect of this type of study will necessarily be related to the assessment, at an elementary level, of the roles played by two of the main extensive magnitudes, internal energy and entropy, in driving the liquid crystalline transitions observed in the fluids under study. We will focus, in particular, on the extension of earlier works that have explored the relationship between statistical entropy and ordering transitions in atomic and molecular fluids by resorting to the multiparticle correlation expansion of entropy established by Green and Nettleton ${ }^{12}$ and later generalized by Lazaridis and co-workers to nonspherical systems. ${ }^{13}$ In this context, many authors have studied the response to ordering in the fluid of the residual multiparticle entropy, defined as the total contributions to the excess entropy of all correlations involving more than two particles. ${ }^{14-17}$ Costa et al. ${ }^{17}$ were the first to employ this methodology to orientational transitions in liquid crystals and found a sudden growth of $\Delta s$ at the isotropic-nematic transition in a HSC fluid. The authors of this latter work imposed a reduced dimensionality in the orientational depen- 


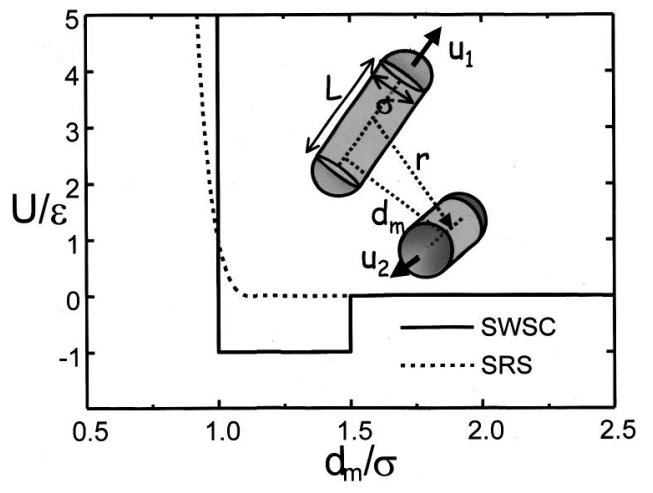

FIG. 1. Pair interaction potential energies as a function of the minimum distance, $d_{m}$, between the molecules for the model fluids studied in this work: square-well spherocylinder fluid [SWSC, see Eq. (1)] and soft repulsive spherocylinder [SRS, see Eq. (2)]. The value of $d_{m}$ depends on the distance between the centers-of-mass of the molecules, $\mathbf{r}$, and on their orientations as defined by the vectors $\mathbf{u}_{\mathbf{1}}$ and $\mathbf{u}_{\mathbf{2}}$.

dence of the radial distribution function and proposed the crossover of $\Delta s$ from negative to positive values as an empirical indication for the incipient transition to the nematic phase, a criterion that had previously been applied to the freezing transition in the hard sphere fluid, ${ }^{14}$ but that showed only moderate success in atomic fluids with attractive interactions. ${ }^{15}$ Thus, in the present study we have addressed the question of whether the entropy criterion, although contrasted for the HSC fluid, does keep its consistency for the liquid crystalline transitions in the SWSC and SRS fluids.

The relevant details of the SWSC and SRS models and of the Monte Carlo simulation methodology employed in this work, including the characterization of the different liquid crystalline phases and the computation of the different structural and thermodynamic magnitudes, are described in Sec. II. The simulation results are presented and discussed thoroughly in Sec. III. Finally, a summary of the main conclusions is drawn in Sec. IV.

\section{SIMULATION METHOD}

\section{A. Model fluids and simulation details}

We have applied the isothermal-isobaric (NPT) version of the Monte Carlo technique to evaluate thermodynamic and structural properties of the SWSC and SRS fluids. In the SWSC fluid, the hard core of the molecules comprises a cylinder of diameter $\sigma$ and elongation $L^{*} \equiv L / \sigma=5$, with hemispherical caps on both ends of the same diameter $\sigma$. The attractive square well interaction is characterized by a depth $\varepsilon$ and a range $\lambda=1.5 \sigma$, and has the same anisotropy of the hard-core. Thus, the pair interaction potential for two such spherocylinders is given by

$$
V(r, \Omega)=\left\{\begin{array}{cc}
\infty & d_{m} \leqslant \sigma \\
-\varepsilon & \sigma<d_{m} \leqslant \lambda \\
0 & d_{m}>\lambda
\end{array}\right.
$$

as represented in Fig. 1. The distance between a pair of molecules, $d_{m}=d_{m}(r, \Omega)$, is defined as the minimum distance between the segments described by the axis of the central cylinder. The value of $d_{m}$ is a function of the relative orien- tation of the molecules, $\Omega$, determined by three independent angles, and of the distance between their centers-of-mass, $r$, as well as of the molecular parameters: diameter and elongation.

In the second model, the SRS fluid, ${ }^{5,9-11}$ the same type of linear molecules interact through a soft and purely repulsive potential of spherocylindrical shape, built from a truncation of a shifted Kihara potential (see Fig. 1) which assures continuity of the potential and its first derivative,

$$
V(r, \Omega)=\left\{\begin{array}{l}
4 \varepsilon\left[\left(\sigma / d_{m}\right)^{12}-\left(\sigma / d_{m}\right)^{6}+1 / 4\right] \quad d_{m} \leqslant \sqrt[6]{2} \cdot \sigma \\
0 \quad d_{m}>\sqrt[6]{2} \cdot \sigma .
\end{array}\right.
$$

The MC-NPT simulations were run at reduced temperature $T^{*} \equiv k T / \varepsilon=5$ ( $k$ denotes the Boltzmann constant) and started at low pressure $\left(P^{*} \equiv P \cdot \sigma^{3} / k T=0.1\right)$ deep in the isotropic region of the phase diagram, over a system of $N_{P}$ $=768$ molecules initially arranged on a hexagonal closepacked (hcp) lattice. Following in part the procedure of McGrother et al., ${ }^{3}$ we built the hcp lattice with the [111] direction along the $z$ axis and a distance between the close packed [111] planes scaled with $\left(1+L^{*}\right)$. The hcp unit cell contains four molecules and we chose a box with 12,8 , and 2 unit cells in the $x, y$, and $z$ directions, respectively. In this way, the dimensions of the simulation box along each axis fulfill the ratios $L_{y} / L_{x}=1.15$ and $L_{z} / L_{x}=1.94$. The longer dimension along the $z$ direction is meant to increase the number of possible smectic layers within the simulation box (up to 4 layers in the close-packed configuration) with respect to the cubic box for a given number of molecules. After equilibration and averaging, the last molecular arrangement is stored and used as initial configuration for the subsequent run with an increased system pressure. Thus, in this way the fluid is compressed through the isotropic-nematic and nematic-smectic transitions. In order to check for possible metastability and hysteric effects, an expansion run, all the way back to the isotropic region, is subsequently performed beginning with the smectic state of highest density in the calculation. As will be discussed below, the expansion run indeed resulted in a better equilibration of the calculations at high density.

The (first-order) liquid crystalline transitions are located by the discontinuities in the density and the order parameter, aided by the analysis of the different correlation functions. The procedure is similar to that employed by McGrother et al. in their study of the HSC fluid ${ }^{3}$ and very recently also by Earl et al. for the SRS fluid. ${ }^{5}$ The nematic order parameter $S \equiv\left\langle P_{2}\left(\mathbf{u}_{\mathbf{i}} \cdot \mathbf{n}\right)\right\rangle$ is calculated as the ensemble average of the mean value of the second Legendre polynomial, its argument being the dot product between the unitary vector along the molecular axis of each particle, $\mathbf{u}_{\mathbf{i}}$, and the (also unitary) nematic director $\mathbf{n}$. For the present study, together with the usual pair distribution function $g(r)$, a relevant orientational distribution function is $g_{2}(r) \equiv\left\langle P_{2}\left(\mathbf{u}_{\mathbf{i}} \cdot \mathbf{u}_{\mathbf{j}}\right)\right\rangle$, defined as the average, for each intermolecular distance, of the second Legendre polynomial with argument the cosine of the relative angle between the axis of particles $i$ and $j, \mathbf{u}_{\mathbf{i}} \cdot \mathbf{u}_{\mathbf{j}}=\cos \theta$. In addition, the translational order of the molecules is further examined by means of the functions $g_{\|}\left(r_{\|}\right)$and $g_{\perp}\left(r_{\perp}\right)$, 
which are the projections of the pair distribution function a distance $r_{\|}$parallel and a distance $r_{\perp}$ perpendicular to the nematic director, respectively. As shown by McGrother et al., ${ }^{3}$ smectic ordering induces well defined oscillations in $g_{\|}\left(r_{\|}\right)$, whereas the structure within the smectic planes is reflected in $g_{\perp}\left(r_{\perp}\right)$. Furthermore, we checked for smectic B hexatic ordering within the smectic layers. We already advance, however, that only smectic A phases were found in the present study.

The thermodynamic (density, energy) and structural properties of the system, as well as the different distribution functions are obtained as ensemble averages in the MC simulation. Typically, for each state an initial simulation of up to $2 \times 10^{6}$ cycles (depending on convergence) is performed to equilibrate the system before averaging for some $1-2 \times 10^{5}$ cycles. Each cycle consists of $N_{P}$ attempts for random displacements and/or reorientations of the particles (whether either or both types of moves are performed is chosen randomly), plus an attempt to change the volume (actually done by rescaling the molecular diameter, $\sigma$, in units of box length). The maximum tilt angle and displacement of the particles and the maximum volume change are adjusted as to give an acceptance ratio of between $30 \%$ and $40 \%$. The usual periodic boundary conditions and minimum image conventions are employed. ${ }^{18}$

\section{B. Entropy criterion for liquid crystalline transitions}

As commented in the Introduction, we have explored the correlation between the configurational entropy and the phase diagram of our liquid crystal models. Following, among others, the works of Lazaridis et al. ${ }^{13}$ and Costa et al. ${ }^{17}$ we have focused on the behavior of the different components of the pair entropy, $s_{2}$, and of the residual entropy, $\Delta s=s_{\mathrm{ex}}-s_{2}$, defined as the multiparticle contribution to the excess entropy, $s_{\mathrm{ex}}$, i.e., all correlations involving more than two particles. As shown in these previous works, ${ }^{13}$ the formal factorization of the correlation function, $g(r, \omega)$ $=g(r) \cdot g(\omega \mid r)$, in terms of $g(r)$, the radial distribution function, and $g(\omega \mid r)$, the conditional distribution function of two molecules with orientations described by $\omega$ (which denotes all the relevant orientation angles) at an intermolecular distance $r$, leads to a decomposition of the pair entropy in orientational and positional contributions.

For the computation of $\Delta s$ in a fluid of linear molecules, Costa $e t$ al. proposed a reduced dimensionality of the correlation function, $g(r, \theta)$, in which the relative orientation of each pair of particles is described by the angle $\theta$ between the two molecular axis, already introduced in Sec. II A. With this assumption, the formal factorization of the correlation function, $g(r, \theta)=g(r) \cdot g(\theta \mid r)$, leads to the following expression for the pair contribution to the excess entropy, $s_{2}$ (per particle and in units of the Boltzmann constant):

$$
\begin{aligned}
& s_{2}=s_{2}^{\mathrm{tr}}+s_{2}^{\mathrm{or}}, \\
& s_{2}^{\mathrm{tr}}=-2 \pi \rho \int[g(r) \ln g(r)-g(r)+1] r^{2} d r,
\end{aligned}
$$

$$
\begin{aligned}
& s_{2}^{\mathrm{or}}=4 \pi \rho \int g(r) S^{\mathrm{or}}(r) r^{2} d r, \\
& S^{\mathrm{or}}(r)=-\frac{1}{4} \int_{0}^{\pi} g(\theta \mid r) \ln g(\theta \mid r) \sin \theta d \theta .
\end{aligned}
$$

In the above expressions (3)-(6), $\rho=N / V$ denotes the number density and $s_{2}^{\text {tr }}$ and $s_{2}^{\text {or }}$ refer to a formal distinction between translational and orientational contributions to $s_{2}$; whereas $s_{2}^{\text {tr }}$ is expected to monitor any changes in the translational order of the molecules of the fluid, $s_{2}^{\text {or }}$ should respond to their orientational order. It must be remarked that the formal decomposition of the pair entropy $s_{2}=s_{2}^{\text {tr }}+s_{2}^{\text {or }}$, as well as Eq. (4) for $s_{2}^{\mathrm{tr}}$, are independent of the approximation introduced by the use of the correlation function $g(r, \theta)$, whose justification relies upon the comparatively simplified computation of $s_{2}^{\text {or }}$, as defined in Eq. (6), and its appropriate behavior when applied to linear nematogens. In fact, Costa et al. found a rapid decrease of $s_{2}^{\text {or }}$ towards large negative values in the vicinity of the isotropic-nematic of the HSC fluid and suggested the zero of the residual entropy, $\Delta s$ $=s_{\text {ex }}-s_{2}=0$, as an indicator for the incipient nematic ordering. 17

The total excess entropy of the isotropic fluid may be evaluated from the MC equation of state, by means of the exact expression,

$$
s_{\mathrm{ex}}(\rho)=\frac{u_{\mathrm{ex}}}{T}-\int_{0}^{\rho}\left[\frac{P}{k T \rho^{\prime}}-1\right] \frac{d \rho^{\prime}}{\rho^{\prime}},
$$

where $u_{\text {ex }}$ denotes the excess energy (i.e., the potential energy) per particle in units of the Boltzmann constant. Hence, the excess entropy is determined by the internal energy and by the integral of $(Z-1) / \rho$, where $Z=P /(k T \rho)$ is the compressibility factor of the fluid.

In the present work, we have extended the computation of the excess entropy to the nematic and smectic phases by including the entropy change at the corresponding phase transitions. Since we are dealing with transitions at constant $N, P, T$, the entropy of transition, $\Delta s_{\text {trans }}$, can be readily obtained from the enthalpy of transition, $\Delta h_{\text {trans }}$, as given by the changes in the internal energy, $\Delta u_{\text {trans }}$, and volume $\Delta v_{\text {trans }}$,

$$
\Delta s_{\text {trans }}=\frac{\Delta h_{\text {trans }}}{T}=\frac{\Delta u_{\text {trans }}}{T}+P^{*} \Delta v_{\text {trans }},
$$

where we recall that $\Delta s_{\text {trans }}, \Delta h_{\text {trans }}, \Delta u_{\text {trans }}$, and $P^{*} \Delta v_{\text {trans }}\left(=P \cdot \Delta v_{\text {trans }} / k T\right)$ are per particle and in $k$ units. Thus, for instance, the extension of Eq. (7) to the calculation of the excess entropy of a particular state after the I-N phase transition is given by

$$
\begin{aligned}
s_{\mathrm{ex}}(\rho)= & \frac{u_{\mathrm{ex}}}{T}-\int_{0}^{\rho_{1}}\left[\frac{P}{k T \rho^{\prime}}-1\right] \frac{d \rho^{\prime}}{\rho^{\prime}}+P_{\mathrm{I}-\mathrm{N}}^{*} \Delta v_{\mathrm{I}-\mathrm{N}} \\
& -\int_{\rho_{2}}^{\rho}\left[\frac{P}{k T \rho^{\prime}}-1\right] \frac{d \rho^{\prime}}{\rho^{\prime}},
\end{aligned}
$$


TABLE I. Isothermal-isobaric Monte Carlo (MC-NPT) simulation results for the equation of state of the SWSC fluid of molecular elongation $L^{*}=L / \sigma=5$, square well depth $\varepsilon$, and range $\lambda^{*}=\lambda / \sigma=1.5$ (see Fig. 1) at temperature $T^{*}=k T / \varepsilon=5$. The pressure, fixed in the simulations, is expressed in reduced units either with respect to the molecular diameter, $\sigma^{3}$ (first column), or to the volume of the molecular hard core, $v_{\text {HSC }}$ (second column), to allow for a direct comparison with earlier works. $U^{*}$ and $S$ denote, respectively, the averages of potential energy per particle and the order parameter of the fluid. The simulations were run by compressing the fluid from the least dense state. The values in brackets denote the statistical uncertainty (one standard deviation) in the last digit. The isotropic (I), nematic $(\mathrm{N})$ or smetic A ( $\mathrm{Sm} \mathrm{A}$ ) phase corresponding for each state is indicated in the last column.

\begin{tabular}{|c|c|c|c|c|c|c|}
\hline$P^{*}=P \cdot \sigma^{3} / k T$ & $P^{*} \cdot v_{\mathrm{HSC}} / \sigma^{3}$ & $\rho^{*}=\rho \cdot \sigma^{3}$ & $\eta=\rho \cdot v_{\mathrm{HSC}}$ & $U^{*}=U / \varepsilon$ & $S$ & Phase \\
\hline 0.01 & 0.045 & $0.0080(3)$ & $0.035(1)$ & $-0.36(2)$ & $0.030(1)$ & I \\
\hline 0.02 & 0.089 & $0.0135(4)$ & $0.060(2)$ & $-0.64(3)$ & $0.030(1)$ & I \\
\hline 0.05 & 0.22 & $0.0245(4)$ & $0.109(2)$ & $-1.25(4)$ & $0.032(1)$ & I \\
\hline 0.10 & 0.45 & $0.0360(4)$ & $0.160(2)$ & $-2.00(4)$ & $0.032(1)$ & I \\
\hline 0.20 & 0.89 & $0.0494(4)$ & $0.220(2)$ & $-2.98(5)$ & $0.033(1)$ & I \\
\hline 0.30 & 1.34 & $0.0580(4)$ & $0.258(2)$ & $-3.66(5)$ & $0.036(1)$ & I \\
\hline 0.40 & 1.78 & $0.0645(4)$ & $0.287(2)$ & $-4.20(5)$ & $0.037(1)$ & I \\
\hline 0.50 & 2.23 & $0.0699(6)$ & $0.311(3)$ & $-4.63(6)$ & $0.042(1)$ & I \\
\hline 0.60 & 2.67 & $0.0741(4)$ & $0.330(2)$ & $-4.97(5)$ & $0.040(1)$ & I \\
\hline 0.70 & 3.12 & $0.0773(4)$ & $0.344(2)$ & $-5.21(5)$ & $0.049(1)$ & I \\
\hline 0.80 & 3.56 & $0.0816(4)$ & $0.363(2)$ & $-5.52(5)$ & $0.042(1)$ & I \\
\hline 0.90 & 4.01 & $0.0845(4)$ & $0.376(2)$ & $-5.69(5)$ & $0.060(1)$ & I \\
\hline 1.00 & 4.45 & $0.0876(4)$ & $0.390(2)$ & $-5.89(5)$ & $0.060(1)$ & I \\
\hline 1.10 & 4.90 & $0.0910(4)$ & $0.405(2)$ & $-6.02(5)$ & $0.072(1)$ & I \\
\hline 1.20 & 5.34 & $0.0932(4)$ & $0.415(2)$ & $-6.13(5)$ & $0.088(2)$ & I \\
\hline 1.25 & 5.56 & $0.0948(4)$ & $0.422(2)$ & $-6.18(5)$ & $0.068(2)$ & I \\
\hline 1.30 & 5.79 & $0.0959(4)$ & $0.427(2)$ & $-6.20(5)$ & $0.115(1)$ & I \\
\hline 1.35 & 6.01 & $0.0980(4)$ & $0.436(2)$ & $-6.21(4)$ & $0.233(1)$ & I \\
\hline 1.40 & 6.23 & $0.0993(4)$ & $0.442(2)$ & $-6.18(6)$ & $0.290(1)$ & I \\
\hline 1.45 & 6.45 & $0.1016(4)$ & $0.452(2)$ & $-6.20(5)$ & $0.486(2)$ & $\mathrm{N}$ \\
\hline 1.50 & 6.68 & $0.1031(4)$ & $0.459(2)$ & $-6.13(5)$ & $0.533(2)$ & $\mathrm{N}$ \\
\hline 1.55 & 6.90 & $0.1047(3)$ & $0.466(1)$ & $-6.08(4)$ & $0.665(2)$ & $\mathrm{N}$ \\
\hline 1.60 & 7.12 & $0.1065(3)$ & $0.474(1)$ & $-6.05(4)$ & $0.719(2)$ & $\mathrm{N}$ \\
\hline 1.65 & 7.34 & $0.1085(4)$ & $0.483(2)$ & $-5.98(4)$ & $0.784(1)$ & $\mathrm{N}$ \\
\hline 1.70 & 7.57 & $0.1132(6)$ & $0.504(3)$ & $-5.79(4)$ & $0.882(2)$ & Sm A \\
\hline 1.75 & 7.79 & $0.1146(4)$ & $0.510(2)$ & $-5.80(5)$ & $0.890(1)$ & Sm A \\
\hline 1.80 & 8.01 & $0.1153(8)$ & $0.513(2)$ & $-5.86(5)$ & $0.852(1)$ & Sm A \\
\hline 1.85 & 8.23 & $0.1180(4)$ & $0.525(2)$ & $-5.81(5)$ & $0.880(2)$ & $\mathrm{Sm} \mathrm{A}$ \\
\hline 1.90 & 8.46 & $0.1202(4)$ & $0.535(2)$ & $-5.77(5)$ & $0.905(3)$ & Sm A \\
\hline 1.95 & 8.68 & $0.1225(6)$ & $0.545(3)$ & $-5.73(5)$ & $0.914(2)$ & Sm A \\
\hline 2.00 & 8.90 & $0.1240(4)$ & $0.552(2)$ & $-5.74(5)$ & $0.915(3)$ & $\mathrm{Sm} \mathrm{A}$ \\
\hline 2.05 & 9.12 & $0.1251(4)$ & $0.557(2)$ & $-5.77(5)$ & $0.906(3)$ & Sm A \\
\hline 2.10 & 9.35 & $0.1263(4)$ & $0.562(2)$ & $-5.81(6)$ & $0.906(4)$ & $\mathrm{Sm} \mathrm{A}$ \\
\hline 2.15 & 9.57 & $0.1272(4)$ & $0.566(2)$ & $-5.82(5)$ & $0.917(3)$ & Sm A \\
\hline 2.20 & 9.79 & $0.1285(4)$ & $0.572(2)$ & $-5.82(5)$ & $0.920(3)$ & Sm A \\
\hline
\end{tabular}

where $\rho_{1}$ and $\rho_{2}$, denote the coexistence densities of the two phases, represented in our NPT simulations by the densities of the states closest on either side to the phase boundary. The transition pressure is taken as the average of the simulation pressure of both boundary states. Note that in Eq. (9) the energetic part of the entropy of transition, $\Delta u_{\mathrm{I}-\mathrm{N}} / T$, is effectively included in $u_{\mathrm{ex}} / T$. The computation of the first integral in Eq. (9) was performed from the Monte Carlo values for $(Z-1) / \rho$ and their linear extrapolation at low density. This latter extrapolation should provide, at $\rho=0$, the second virial coefficient, $B_{2}$, of the fluid at the title temperature. In fact, as shown below (Fig. 7), the extrapolation to $\rho=0$ of the computed $(Z-1) / \rho$ curve for the SWSC system is consistent with the known analytical expression of $B_{2}$ for this model fluid. ${ }^{6}$

We have employed Eqs. (3)-(9) to extend the study of the behavior of $s_{\mathrm{ex}}, \Delta s$ and the different components of $s_{2}$, through both the isotropic-nematic and the nematic-smectic transitions of the SWSC and SRS fluids.

\section{RESULTS AND DISCUSSION}

We have performed MC-NPT simulations for the SWSC and SRS fluids of elongation $L^{*}=5$ at a reduced temperature $T^{*}=5$. The thermodynamic data resulting in the compression and expansion simulation runs for this isotherm of both model fluids are listed in Tables I-IV. In addition, the equations of state $\left(P^{*}\right.$ vs $\left.\rho^{*}\right)$, order parameters and internal energies obtained for both models are represented in Figs. 2, 4, and 6. Within the range of pressures covered in our study, $P^{*} \approx 0.1-2.2$, both the SWSC and the SRS fluids exhibit differentiated isotropic, nematic, and smectic A phases. The 
TABLE II. Same as Table I, but for a set of simulations run by expanding the SWSC fluid from the most dense state $\left(P^{*}=2.20\right)$.

\begin{tabular}{ccccccc}
\hline \hline$P^{*}=P \cdot \sigma^{3} / k T$ & $P^{*} \cdot v_{\mathrm{HSC}} / \sigma^{3}$ & $\rho^{*}=\rho \cdot \sigma^{3}$ & $\eta=\rho \cdot v_{\mathrm{HSC}}$ & $U^{*}=U / \varepsilon$ & $S$ & Phase \\
\hline 1.00 & 4.45 & $0.0881(6)$ & $0.392(3)$ & $-5.87(7)$ & $0.085(1)$ & $\mathrm{I}$ \\
1.10 & 4.90 & $0.0905(4)$ & $0.403(2)$ & $-6.03(5)$ & $0.084(1)$ & $\mathrm{I}$ \\
1.20 & 5.34 & $0.0930(4)$ & $0.414(2)$ & $-6.11(5)$ & $0.146(2)$ & $\mathrm{I}$ \\
1.25 & 5.56 & $0.0953(6)$ & $0.424(3)$ & $-6.15(5)$ & $0.175(2)$ & $\mathrm{I}$ \\
1.30 & 5.79 & $0.0968(6)$ & $0.431(3)$ & $-6.17(5)$ & $0.215(2)$ & $\mathrm{I}$ \\
1.35 & 6.01 & $0.0993(4)$ & $0.442(2)$ & $-6.02(5)$ & $0.557(2)$ & $\mathrm{N}$ \\
1.40 & 6.23 & $0.1007(4)$ & $0.448(2)$ & $-6.04(5)$ & $0.609(2)$ & $\mathrm{N}$ \\
1.45 & 6.45 & $0.1027(8)$ & $0.457(4)$ & $-5.98(6)$ & $0.691(2)$ & $\mathrm{N}$ \\
1.50 & 6.68 & $0.1043(6)$ & $0.464(3)$ & $-5.99(5)$ & $0.716(2)$ & $\mathrm{N}$ \\
1.55 & 6.90 & $0.1058(6)$ & $0.471(3)$ & $-5.97(5)$ & $0.772(3)$ & $\mathrm{N}$ \\
1.60 & 7.12 & $0.1078(6)$ & $0.480(3)$ & $-6.00(5)$ & $0.789(3)$ & $\mathrm{N}$ \\
1.65 & 7.34 & $0.1099(5)$ & $0.489(3)$ & $-5.92(5)$ & $0.824(3)$ & $\mathrm{N}$ \\
1.70 & 7.57 & $0.1155(6)$ & $0.514(3)$ & $-5.67(3)$ & $0.907(3)$ & Sm A \\
1.75 & 7.79 & $0.1171(5)$ & $0.521(3)$ & $-5.70(3)$ & $0.913(3)$ & Sm A \\
1.80 & 8.01 & $0.1189(4)$ & $0.529(2)$ & $-5.70(5)$ & $0.911(3)$ & Sm A \\
1.90 & 8.46 & $0.1218(4)$ & $0.542(2)$ & $-5.72(4)$ & $0.915(3)$ & Sm A \\
2.00 & 8.90 & $0.1243(6)$ & $0.553(3)$ & $-5.80(5)$ & $0.915(3)$ & Sm A \\
2.10 & 9.35 & $0.1263(4)$ & $0.562(2)$ & $-5.81(5)$ & $0.926(3)$ & Sm A \\
2.20 & 9.79 & $0.1285(4)$ & $0.572(2)$ & $-5.82(5)$ & $0.920(3)$ & Sm A \\
\hline \hline
\end{tabular}

phase transitions involve relatively weak discontinuous changes in density (see Fig. 6) and internal energy but are accompanied by appreciable jumps in the order parameter and/or in the structure of the different radial correlation func- tions, $g(r), g_{2}(r)$, and in $g_{\|}\left(r_{\|}\right)$, defined above and depicted in Figs. 3 and 5. As we shall see below, the pair and residual entropies also respond to the orientational and translational ordering of the fluid (Figs. 7 and 8).

TABLE III. Isothermal-isobaric Monte Carlo (MC-NPT) simulation results for the equation of state of the SRS fluid [Eq. (2), Fig. 1] of molecular elongation $L^{*}=L / \sigma=5$ at temperature $T^{*}=k T / \varepsilon=5$. The simulations were run by compressing the fluid from the least dense state. The same notation is employed as in Table I. In particular, $v_{\mathrm{HSC}}$ denotes the volume of a hard spherocylinder with the same elongation $L^{*}=5$.

\begin{tabular}{|c|c|c|c|c|c|c|}
\hline$P^{*}=P \cdot \sigma^{3} / k T$ & $P^{*} \cdot v_{\mathrm{HSC}} / \sigma^{3}$ & $\rho^{*}=\rho \cdot \sigma^{3}$ & $\eta=\rho \cdot v_{\mathrm{HSC}}$ & $U^{*}=U / \varepsilon$ & $S$ & Phase \\
\hline 0.01 & 0.044 & $0.0079(4)$ & $0,035(2)$ & $0.12(3)$ & $0.031(1)$ & I \\
\hline 0.02 & 0.089 & $0.0134(4)$ & $0,060(2)$ & $0.23(4)$ & $0.030(1)$ & I \\
\hline 0.05 & 0.22 & $0.0249(4)$ & $0,111(2)$ & $0.48(6)$ & $0.031(1)$ & I \\
\hline 0.10 & 0.45 & $0.0368(4)$ & $0.164(2)$ & $0.83(7)$ & $0.033(1)$ & I \\
\hline 0.20 & 0.89 & $0.0521(6)$ & $0.232(3)$ & $1.4(1)$ & $0.035(1)$ & I \\
\hline 0.30 & 1.34 & $0.0627(6)$ & $0.279(3)$ & $1.9(1)$ & $0.037(1)$ & I \\
\hline 0.40 & 1.78 & $0.0708(6)$ & $0.315(3)$ & $2.4(1)$ & $0.040(1)$ & I \\
\hline 0.50 & 2.23 & $0.0775(6)$ & $0.345(3)$ & $2.9(1)$ & $0.040(1)$ & I \\
\hline 0.70 & 3.12 & $0.0887(6)$ & $0.395(3)$ & $3.7(1)$ & $0.048(1)$ & I \\
\hline 0.80 & 3.56 & $0.0937(6)$ & $0.417(3)$ & $4.1(1)$ & $0.055(1)$ & I \\
\hline 0.90 & 4.01 & $0.0982(6)$ & $0.437(3)$ & $4.5(1)$ & $0.061(1)$ & I \\
\hline 1.00 & 4.45 & $0.1025(6)$ & $0.456(3)$ & $4.8(1)$ & $0.071(1)$ & I \\
\hline 1.10 & 4.90 & $0.1065(6)$ & $0.474(3)$ & $5.2(2)$ & $0.134(2)$ & I \\
\hline 1.15 & 5.12 & $0.1088(8)$ & $0.484(4)$ & $5.4(2)$ & $0.110(3)$ & I \\
\hline 1.20 & 5.34 & $0.1117(6)$ & $0.497(3)$ & $5.5(2)$ & $0.198(2)$ & I \\
\hline 1.25 & 5.56 & $0.1157(6)$ & $0.515(3)$ & $5.7(2)$ & $0.541(2)$ & $\mathrm{N}$ \\
\hline 1.30 & 5.79 & $0.1200(8)$ & $0.534(4)$ & $5.8(2)$ & $0.722(2)$ & $\mathrm{N}$ \\
\hline 1.35 & 6.01 & $0.1216(8)$ & $0.541(4)$ & $6.0(2)$ & $0.715(2)$ & $\mathrm{N}$ \\
\hline 1.40 & 6.23 & $0.1240(8)$ & $0.552(4)$ & $6.2(2)$ & $0.752(2)$ & $\mathrm{N}$ \\
\hline 1.45 & 6.45 & $0.1258(8)$ & $0.560(4)$ & $6.3(2)$ & $0.766(2)$ & $\mathrm{N}$ \\
\hline 1.50 & 6.68 & $0.1281(8)$ & $0.570(4)$ & $6.5(2)$ & $0.795(2)$ & $\mathrm{N}$ \\
\hline 1.55 & 6.90 & $0.1303(8)$ & $0.580(4)$ & $6.6(2)$ & $0.820(3)$ & $\mathrm{N}$ \\
\hline 1.60 & 7.12 & $0.1319(8)$ & $0.587(4)$ & $6.8(2)$ & $0.826(4)$ & $\mathrm{N}$ \\
\hline 1.65 & 7.34 & $0.1339(8)$ & $0.596(4)$ & $7.0(2)$ & $0.838(3)$ & $\mathrm{N}$ \\
\hline 1.70 & 7.57 & $0.1360(8)$ & $0.605(4)$ & $7.1(2)$ & $0.855(3)$ & $\mathrm{N}$ \\
\hline 1.75 & 7.79 & $0.1370(8)$ & $0.610(4)$ & $7.3(2)$ & $0.862(3)$ & $\mathrm{N}$ \\
\hline 1.80 & 8.01 & $0.1398(8)$ & $0.622(4)$ & $7.4(2)$ & $0.880(4)$ & $\mathrm{N}$ \\
\hline 1.85 & 8.23 & $0.145(1)$ & $0.644(5)$ & $7.4(2)$ & $0.915(3)$ & $\mathrm{N}$ \\
\hline 1.90 & 8.46 & $0.147(1)$ & $0.655(5)$ & $7.5(2)$ & $0.920(2)$ & $\mathrm{Sm} \mathrm{A}$ \\
\hline 1.95 & 8.68 & $0.151(1)$ & $0.673(6)$ & $7.5(2)$ & $0.937(2)$ & $\mathrm{Sm} \mathrm{A}$ \\
\hline 2.00 & 8.90 & $0.153(1)$ & $0.681(5)$ & $7.6(2)$ & $0.940(3)$ & $\mathrm{Sm} \mathrm{A}$ \\
\hline 2.10 & 9.35 & $0.158(1)$ & $0.702(5)$ & $7.9(2)$ & $0.950(3)$ & $\mathrm{Sm} \mathrm{A}$ \\
\hline
\end{tabular}


TABLE IV. Same as Table III, but for a set of simulations run by expanding the SRS fluid from the most dense state $\left(P^{*}=2.10\right)$.

\begin{tabular}{ccccccc}
\hline \hline$P^{*}=P \cdot \sigma^{3} / k T$ & $P^{*} \cdot v_{\mathrm{HSC}} / \sigma^{3}$ & $\rho^{*}=\rho \cdot \sigma^{3}$ & $\eta=\rho \cdot v_{\mathrm{HSC}}$ & $U^{*}=U / \varepsilon$ & $S$ & Phase \\
\hline 1.00 & 4.45 & $0.1025(6)$ & $0.456(3)$ & $4.8(1)$ & $0.058(2)$ & $\mathrm{I}$ \\
1.10 & 4.90 & $0.1067(6)$ & $0.475(3)$ & $5.2(2)$ & $0.096(2)$ & $\mathrm{I}$ \\
1.20 & 5.34 & $0.1119(8)$ & $0.498(4)$ & $5.5(2)$ & $0.267(2)$ & $\mathrm{I}$ \\
1.25 & 5.56 & $0.1157(8)$ & $0.515(4)$ & $5.7(2)$ & $0.504(2)$ & $\mathrm{N}$ \\
1.30 & 5.79 & $0.1195(8)$ & $0.532(4)$ & $5.8(2)$ & $0.698(2)$ & $\mathrm{N}$ \\
1.35 & 6.01 & $0.1220(8)$ & $0.543(4)$ & $6.0(2)$ & $0.722(2)$ & $\mathrm{N}$ \\
1.40 & 6.23 & $0.1240(8)$ & $0.552(4)$ & $6.2(2)$ & $0.740(2)$ & $\mathrm{N}$ \\
1.50 & 6.68 & $0.1285(8)$ & $0.572(4)$ & $6.5(2)$ & $0.802(2)$ & $\mathrm{N}$ \\
1.60 & 7.12 & $0.1323(8)$ & $0.589(4)$ & $6.8(2)$ & $0.830(2)$ & $\mathrm{N}$ \\
1.65 & 7.34 & $0.1343(8)$ & $0.598(4)$ & $7.0(2)$ & $0.823(3)$ & $\mathrm{N}$ \\
1.70 & 7.57 & $0.1371(8)$ & $0.610(4)$ & $7.1(2)$ & $0.864(3)$ & $\mathrm{N}$ \\
1.75 & 7.79 & $0.1387(8)$ & $0.617(4)$ & $7.3(2)$ & $0.879(3)$ & $\mathrm{N}$ \\
1.80 & 8.01 & $0.1431(8)$ & $0.637(5)$ & $7.4(2)$ & $0.904(3)$ & $\mathrm{Sm} \mathrm{A}$ \\
1.85 & 8.23 & $0.1465(8)$ & $0.652(5)$ & $7.5(2)$ & $0.931(3)$ & Sm A \\
1.90 & 8.46 & $0.1492(8)$ & $0.664(6)$ & $7.7(2)$ & $0.938(3)$ & Sm A \\
2.00 & 8.90 & $0.1557(8)$ & $0.693(6)$ & $7.8(2)$ & $0.953(3)$ & Sm A \\
2.10 & 9.35 & $0.1577(8)$ & $0.702(5)$ & $7.9(2)$ & $0.950(3)$ & Sm A \\
\hline \hline
\end{tabular}

\section{A. Phase diagram of the SWSC fluid}

We focus now on the discussion of the simulation results for the SWSC fluid. The isotherm obtained by compressing the system from the isotropic phase (Table I and Fig. 2) evolves smoothly with increasing pressure up to $P^{*} \approx 1.30$. In this low pressure interval, both the density and the absolute value of the potential energy become progressively larger, while the order parameter remains small $(S<0.1)$ and roughly constant. At $P^{*}=1.35-1.40$, the number density is sufficiently high and close to the incipient isotropic-nematic transition as to induce fluctuations in the system that take the order parameter above 0.2. At the same time, the internal energy approaches an extremum and stabilizes at $U^{*}$ $\approx-6.2$. In fact, at $P^{*}=1.45$ the order parameter jumps to $S=0.486$ and enters already the nematic phase of the fluid; this is the first state included in the compression run that displays long-range orientational order, as monitored by $g_{2}(r)$. The density, internal energy and order parameter of this first nematic state, as well as the behavior of the thermodynamic variables in the proximity of the transition, are consistent with the result of similar simulations of Williamson and del Río ${ }^{6}$ performed over a larger number of particles $\left(N_{P}=1020\right)$.

Within the nematic phase, the further increase of pressure and density of the SWSC fluid induces a greater orientational ordering which takes the order parameter to $S>0.7$ for $P^{*}>1.55$. At the same time the potential energy becomes slightly less negative (i.e., the energy increases). The density, energy and order parameter maintain their increasing trend with growing $P^{*}$, and at $P^{*}=1.70$ the translational ordering characteristic of a smectic A phase develops and persists in the remaining higher pressure states included in our study. No evidence for smectic B or a solid phase order within each of the smectic layers was detected, which is not unexpected, since for the HSC fluid of same elongation the smectic

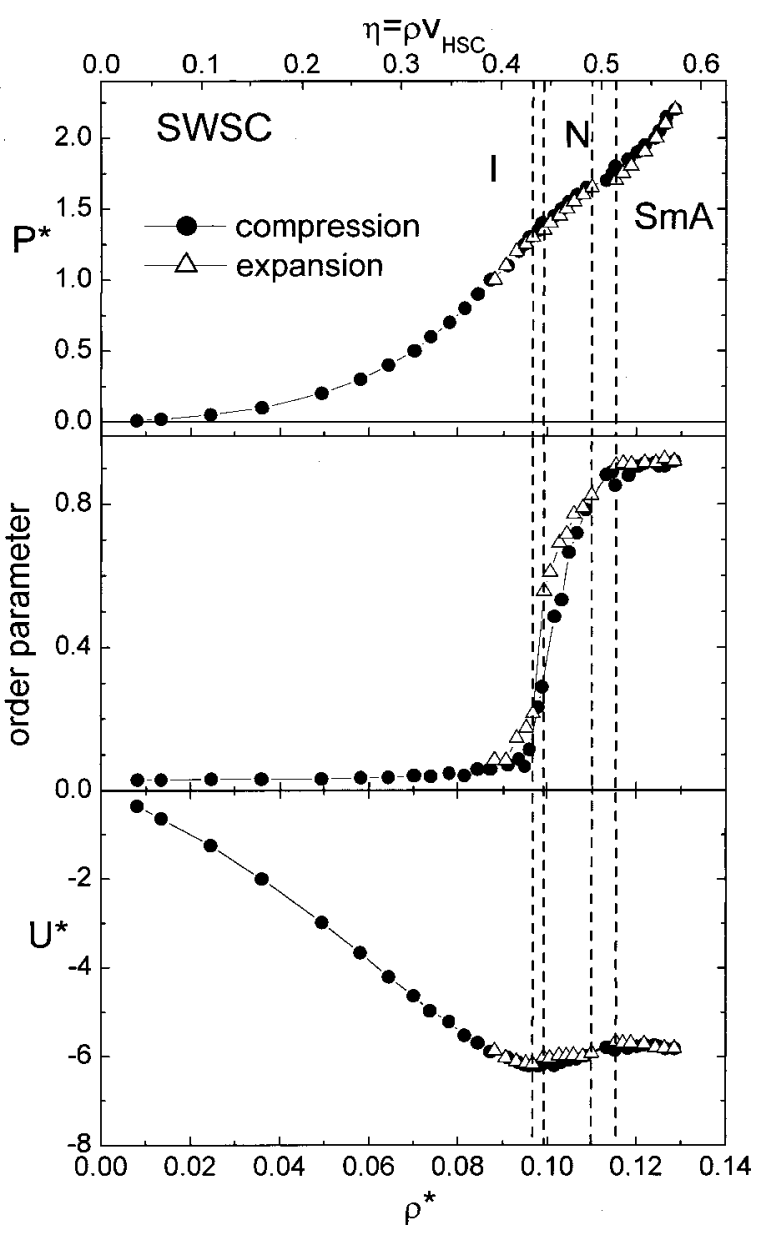

FIG. 2. MC-NPT results for the equation of state (top), order parameter (middle), and internal energy per particle (bottom) for the SWSC fluid model. Solid circles and open triangles denote the simulation series run by compressing and expanding the fluid, respectively (see text). The vertical dashed lines indicate the coexistence densities at the isotropic-nematic (I-N) and at the nematic-smectic A (N-Sm A) phase transitions, obtained in the simulations when expanding the fluid (see Table V). 
A-solid transition is observed at significantly higher pressure $\left(P^{*} \approx 2.5\right)$ and density $\left(\rho^{*} \approx 0.138\right) .{ }^{3}$

As mentioned above, in order to assess the stability of the different phases observed in the simulations, an expansion run was performed from the smectic state of highest pressure $\left(P^{*}=2.20\right)$ back to the isotropic region $\left(P^{*}\right.$ $=1.00$ ). The relevant data concerning the expansion simulation set are listed in Table II and also shown in Fig. 2. As the system pressure is decreased in this expansion run, the fluid undergoes the reverse sequence of phase transitions $\mathrm{Sm} \mathrm{A}-\mathrm{N}-\mathrm{I}$ and a moderate hysteresis is observed. The isotropic-nematic and the nematic-smectic A phase boundaries are in this case located within the intervals $\left(P^{*}\right.$ $\left.=1.30-1.35, \rho^{*}=0.0968-0.0993\right)$ and $\left(P^{*}=1.65-1.70\right.$, $\rho^{*}=0.1099-0.1155$ ), respectively (see Table V), and, hence, appear slightly shifted toward smaller pressures and/or densities in comparison to the compression run discussed in the precedent paragraphs. Similar effects were observed in the simulations of Williamson and del Río for the isotropic-nematic transition of this same fluid. ${ }^{6}$ Since the equilibration of an ordered state from an initial disordered configuration is more demanding than the reverse case when performing MC simulations, we conclude that, in the compression run, the states of higher density within the isotropic and nematic phases are actually metastable, i.e., unstable with respect to the more ordered nematic and smectic phases, respectively. Therefore, when discussing the liquid crystalline behavior of the SWSC fluid in the remaining of the paper, we will consider solely the results arising from the simulations of the expansion run.

Figure 3 represents the radial functions $g(r), g_{2}(r)$, and $g_{\|}\left(r_{\|}\right)$(see above for definitions) for relevant states of the expansion MC run for the SWSC fluid. It is remarkable, for instance, how $g_{2}(r)$ clearly reflects, for $P^{*} \geqslant 1.35$, the long range orientational order that differentiates qualitatively the nematic and smectic phases from the isotropic one. On the other hand, the smectic order that characterizes the states at $P^{*} \geqslant 1.70$ in the expansion run is clearly observed not only in $g_{\|}\left(r_{\|}\right)$but also in the radial distribution function $g(r)$. As can be seen in Fig. 3, with growing density all through the isotropic and nematic phases, $g(r)$ develops progressively more differentiated maxima associated to the first and second neighboring molecules (at $r / \sigma \approx 1.1$ and 2.3 , respectively). However, after the nematic-smectic A transition (i.e., from $P^{*}=1.65$ to $\left.P^{*}=1.70\right)$, the structure of $g(r)$ changes qualitatively, with a sudden increase of the area of the first, second, and even third, next-neighbor peaks, and the appearance of a depression at $r / \sigma \approx 3.5-6.0$, where $g(r)$ stays below unity, as a consequence of the layered structure of the fluid. It must be noted, however, that, due to the weak first-order character of the liquid crystal transitions presently studied, and in spite of the qualitative changes undergone by $g(r)$, $g_{2}(r)$, and $g_{\|}\left(r_{\|}\right)$at the I-N and N-Sm A phase transitions, it is not necessarily straightforward to assign the boundary states for each transition in $\mathrm{MC}$ simulations unavoidably performed over a finite number of particles. For instance, the assignment of the $\mathrm{N}-\mathrm{Sm} \mathrm{A}$ transition, based on the observation of an appreciable jump in density (see Fig. 6) accompanied by a simultaneous jump in the amplitude of the layering
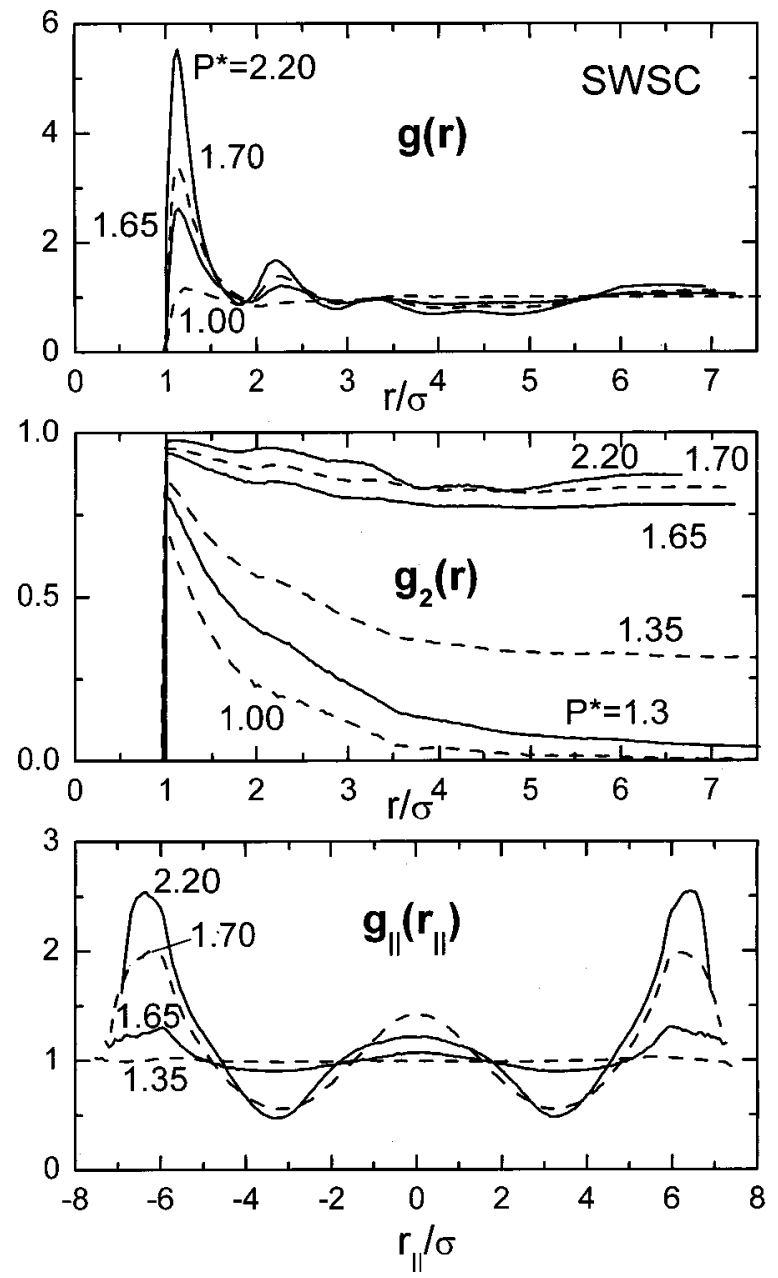

FIG. 3. Correlation functions for representative states of the SWSC fluid in the present study (see Sec. II A for definitions). The system pressure of each state is indicated next to the corresponding curve. (Top) radial distribution function $g(r) ; \quad$ (middle) orientational distribution function $g_{2}(r)$ $\equiv\left\langle P_{2}(\cos \theta)\right\rangle$; (bottom) projection of the pair distribution function a distance $r_{\|}$parallel to the nematic director $g_{\|}\left(r_{\|}\right)$. Oscillations in this latter function are indicative of layered smectic ordering in the fluid.

maxima in $g_{\|}\left(r_{\|}\right)$and in the structure of $g(r)$, leaves a weak onset of layering $\left[\approx 1.2\right.$ amplitude in $\left.g_{\|}\left(r_{\|}\right)\right]$in the state of highest density considered nematic in our study $\left(P^{*}=1.65\right.$ in the SWSC fluid, $P^{*}=1.75$ in the SRS fluid, see bottom of Figs. 3 and 5). This same effect was noted by McGrother et al. in their study of the HSC fluid. ${ }^{3}$ It seems also timely to comment on the apparent more pronounced oscillations of $g_{\|}\left(r_{\|}\right)$at the edge of the simulation cell, whereas the correct structure of this function expected in the smectic phase would be a sequence of maxima of the same height. However, due to the limited number of particles employed in our simulations, in the computation of $g_{\|}\left(r_{\|}\right)$the first neighboring smectic layers are not sampled as efficiently as the central layer, and thus the computed maxima at $\left|r_{\|} / \sigma\right| \approx 6.5$ are too narrow and overestimated in height. Simulations with a larger number of particles (and, thus, an increased size of the simulation cell), as those of Ref. 3 for the HSC system with $L^{*}=5$, largely correct for this effect.

Summarizing, the pressures and coexistence densities for the isotropic-nematic and nematic-smectic A transitions for 
the SWSC fluid, as obtained by averaging the pressures of the two boundary states of each phase transition (in the expansion $\mathrm{MC}$ run) and from their individual densities, are $P_{\mathrm{I}-\mathrm{N}}^{*}=1.325 ; \rho_{\mathrm{I}}^{*}=0.0968, \rho_{\mathrm{N}}^{*}=0.0993$, and $P_{\mathrm{N}-\mathrm{Sm} \mathrm{A}}^{*}$ $=1.675 ; \quad \rho_{\mathrm{N}}^{*}=0.1109, \quad \rho_{\mathrm{Sm} \mathrm{A}}^{*}=0.1155$, respectively (see Table V).

The specific role of the square well in the liquid crystal behavior of the SWSC fluid may be assessed by contrasting the present results with the MC-NPT simulations of McGrother et al. for the hard spherocylinder HSC fluid. ${ }^{3} \mathrm{We}$ begin by recalling the remarkable stabilization of the internal energy of the SWSC fluid at roughly constant values around $U^{*}=-6.0$ at high density $\left(\rho^{*}>0.09\right)$ (lower panel of Fig. 2 ). Since such stabilization takes place right before the I-N transition, it might be tentatively concluded that the energetic contribution to the free energy of the system "saturates" and has a negligible effect in the liquid crystalline behavior of the fluid. In fact, such a saturation effect may be understood as a direct consequence of the square-well interaction imposed in the model: at high enough densities all nearest-neighbors are at distance closer than the SW range and thus move freely inside the square well without change of energy. ${ }^{19}$ Under this interpretation it follows that the SWSC fluid should virtually resemble the liquid crystalline behavior of the HSC fluid.

However, we find that both the $\mathrm{I}-\mathrm{N}$ and the $\mathrm{N}-\mathrm{Sm} \mathrm{A}$ phase transitions of the SWSC fluid are delayed toward higher densities and pressures with respect to the HSC fluid. This can be seen in Fig. 6 which depicts in more detail the equation of state for the HSC and the SWSC fluids in the vicinity of the I-N and the $\mathrm{N}-\mathrm{Sm}$ A transitions. The corresponding transition pressures and densities are compared in Table V. For the HSC system, the I-N and N-Sm A transitions were determined to be within $\left(P_{\mathrm{I}-\mathrm{N}}^{*}=1.19\right.$, $\left.\rho_{\mathrm{I}-\mathrm{N}}^{*}=0.0914-0.0932\right)$ and $\left(P_{\mathrm{N}-\mathrm{Sm} \mathrm{A}}^{*}=1.540, \rho_{\mathrm{N}-\mathrm{Sm} \mathrm{A}}^{*}\right.$ $=0.1061-0.1095)$, respectively. ${ }^{3}$ Thus, the energetic contribution of the attractive well to the free energy does seem to drive partly these ordering transitions. The presence of the square well imposes configurational constrains that stabilize the isotropic phase with respect to the nematic phase, and also this latter one with respect to the smectic A phase. It follows that the role of the internal energy opposes in this case that of the main driving force of the liquid crystalline transitions; the configurational entropy, a magnitude that is controlled by excluded volume effects (the only relevant effect for the HSC fluid), mainly a competition between the phase space accessible for the rotation and translation of the molecules. We finally note that for arbitrarily high temperatures (i.e., $T \rightarrow \infty$ ) the phase diagram of the SWSC system should tend asymptotically to that of the HSC fluid of same elongation. Hence, with increasing temperature the location of the liquid crystal transitions of the former are expected to shift smoothly toward smaller pressures and densities.

\section{B. Phase diagram of the SRS fluid}

We concentrate now on the simulation results for the SRS fluid. The isotherms obtained when compressing the fluid from the isotropic phase (Table III and Fig. 4) or expanding the system back from the smectic phase (Table IV

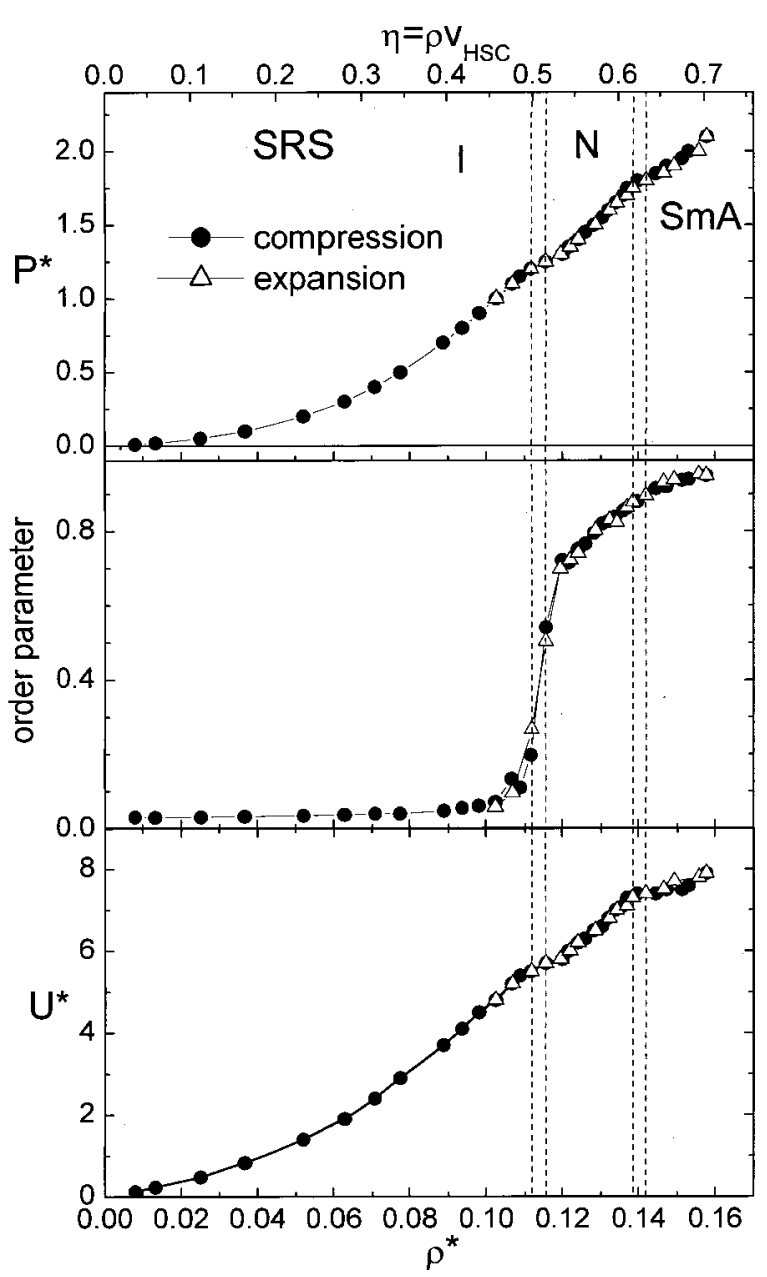

FIG. 4. MC-NPT results for the equation of state (top), order parameter (middle), and internal energy per particle (bottom) for the SRS fluid model. The same notation as in Fig. 2 is used. The isotropic-nematic (I-N) and the namatic-smectic A ( N-Sm A) coexistence densities are indicated by vertical dashed lines (see Table V). Note that, in spite of the soft core of the SRS fluid [Eq. (2)], the packing fraction given in the upper axis (for a more direct comparison with earlier works) is defined with respect to $v_{\mathrm{HSC}}$, the volume of a hard spherocylinder of the same elongation $L^{*}=5$.

and Fig. 4) display a sequence of isotropic-nematic-smectic A phases involving the same qualitative behavior in the relevant structure parameters and correlation functions (Fig. 5) as that observed for the SWSC system. The simulations for the SRS fluid showed a weaker hysteresis than the SWSC fluid, yielding more similar results in the compression and expansion runs. However, some degree of hysteresis is still appreciable and we will refer to the results of the expansion run in the following discussion of the liquid crystalline properties of the SRS fluid.

There are quite significant quantitative differences between the SRS system with respect to the SWSC and HSC models. As can be readily observed in Figs. 4 and 6, for a given pressure, the soft core of the SRS interaction brings the fluid to considerably larger densities in comparison to the HSC (Ref. 3) and the SWSC fluids. In addition, with respect to these latter systems, the SRS fluid presents I-N and N-Sm A transitions under quite different pressure/ density conditions, namely within the intervals $\left(P_{\mathrm{I}-\mathrm{N}}^{*}=1.20-1.25, \rho_{\mathrm{I}-\mathrm{N}}^{*}=0.1119-0.1157\right)$ and $\left(P_{\mathrm{N}-\mathrm{Sm} A}^{*}\right.$ 

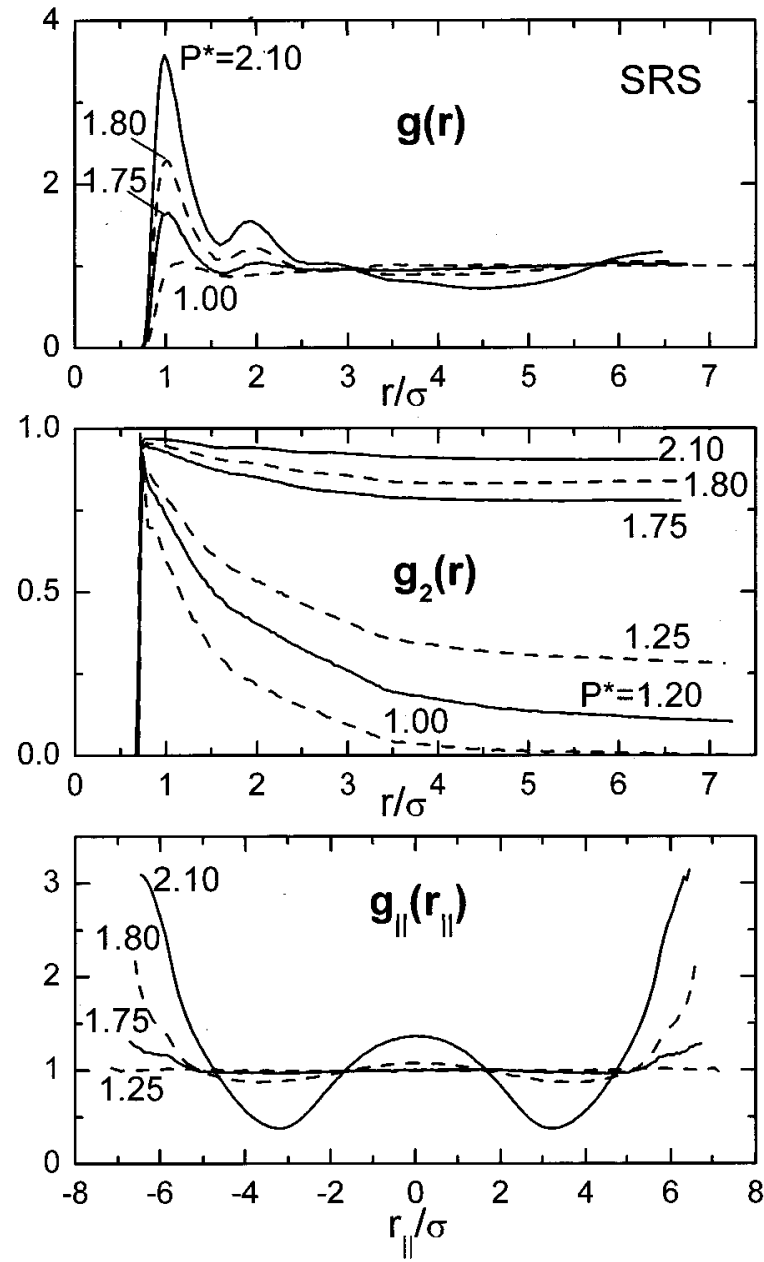

FIG. 5. Correlation functions for representative states of the SRS fluid in the present study (see Fig. 3 and Sec. II A for definitions). The system pressure of each state is indicated next to each curve.

$=1.75-1.80, \rho_{\mathrm{N}-\mathrm{Sm} \mathrm{A}}^{*}=0.1386-0.1420$ ), respectively (see Table V and Fig. 6). It is interesting to note in particular that, although the SRS nematic phase becomes stable at pressures only slightly higher than the HSC fluid and slightly lower than the SWSC fluid, the SRS isotropic-nematic transition takes place at considerably higher densities. It becomes apparent that the soft core of the spherocylinders allows for a significantly greater packing of the molecules in the isotropic phase (before the appearance of the nematic phase) in comparison to their hard core counterparts. In fact, the density of the highest isotropic state of the SRS fluid in our simulations $\left(\rho^{*}=0.1119\right)$ lies already very close to the coexistence densities at the N-Sm A transition of the SWSC fluid ( $\rho^{*}$ $=0.1109-0.1155)$. An even more remarkable effect in this same direction takes place within the nematic phase of the SRS fluid, which remains stable with respect to the smectic phase over a broader pressure/density range and up to considerably higher densities than for the HSC and SWSC fluids. We again refer the reader to Fig. 6 and Table V for a global comparison between the phase diagrams of the HSC, SWSC, and SRS fluids.

The stabilization of the isotropic phase with respect to the nematic phase and, more remarkably, of this latter phase with respect to the smectic A phase, when substituting the
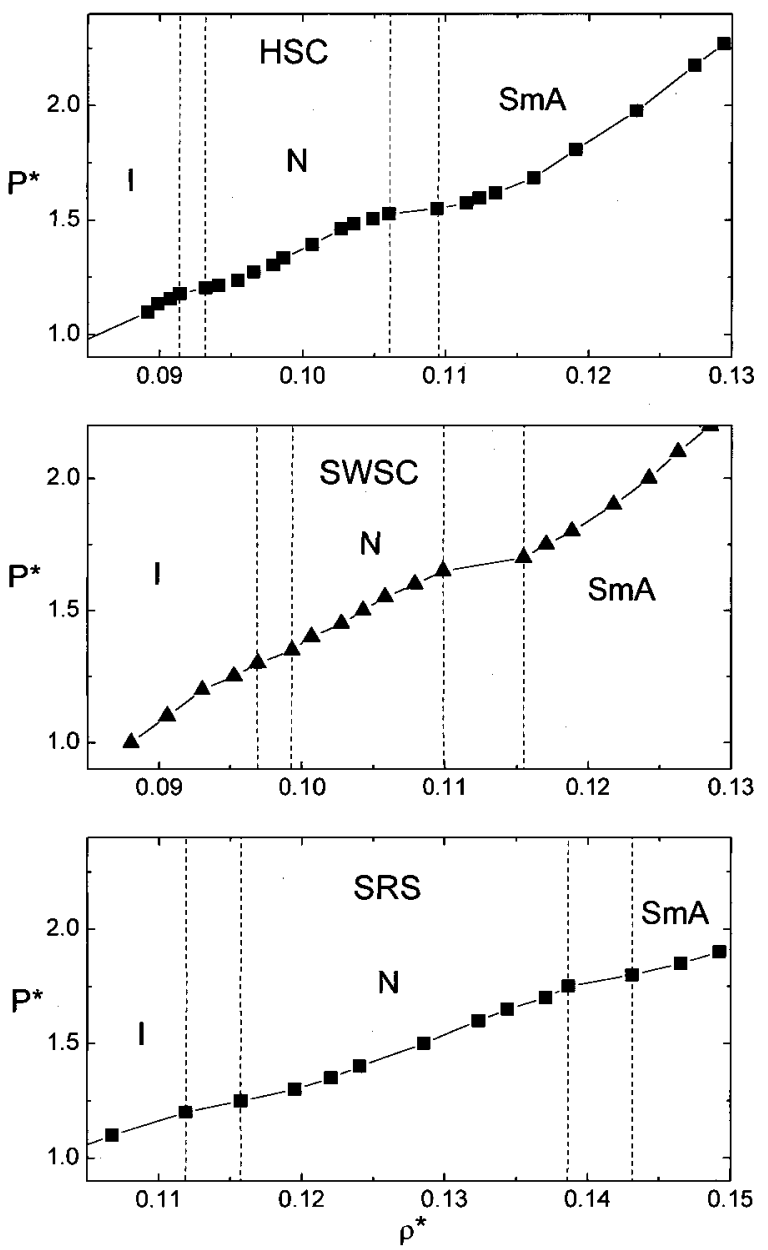

FIG. 6. Detail of the equations of state $\left(P^{*}\right.$ vs $\left.\rho^{*}\right)$ of the HSC (Ref. 3), SWSC, and SRS fluids in the vicinity of the isotropic-nematic (I-N) and the nematic-smectic A $(\mathrm{N}-\mathrm{Sm} \mathrm{A})$ transitions. Note that the density scale (horizontal axis) in the bottom panel (SRS fluid) is displaced with respect to the top (HSC fluid) and the middle (SWSC fluid) panels.

HSC or SWSC interactions by the SRS one, can be directly attributed to the lesser relevance in the SRS fluid of entropic excluded volume effects. The soft core molecules are able to explore a much greater volume of the phase space in comparison to their hard-core counterparts, whereas molecular overlaps are forbidden altogether in the HSC and SWSC fluids, they are allowed in the SRS fluid at the expense of an increase in the internal energy. In this context, it is interesting to note that the average potential energy of the SRS system increases monotonously with density, but with a smaller slope after each liquid crystalline phase change. Thus, such transitions are beneficial from a purely energetic point of view, but do not take place until the dominant entropic contribution is favored.

We notice at this point the strong contrast of our present results with the study of Earl et al. for the SRS fluid with $L^{*}=4$ and $T^{*}=1 .^{5}$ The NPT-MC simulations for this latter fluid yielded $\mathrm{I}-\mathrm{N}$ and $\mathrm{N}-\mathrm{Sm}$ A phase transitions located in each case at pressures and densities significantly smaller than for the HSC fluid of same elongation. The study therefore concluded, in clear opposition to our results, that the SRS interaction stabilizes the nematic phase with respect to the isotropic phase but destabilizes it with respect to the smectic 
TABLE V. Coexistence pressures and densities for the isotropic nematic (I-N) and for the nematic-smectic A (N-Sm A) transitions of the SWSC and the SRS fluids. The tabulated densities correspond to the boundary states for each transition (i.e., consecutive states in the NPT-MC simulation belonging to different phases), whereas the coexistence pressure is obtained from the mean value of the simulation pressures of the two boundary states. The coexistence parameters obtained in the expansion MC runs (indicated in boldface in the table) are considered to be more accurate than those of the compressing runs (see text). Similar results for the HSC fluid from Ref. 3 are also included for comparison.

\begin{tabular}{|c|c|c|c|c|c|c|}
\hline & \multicolumn{3}{|c|}{$\mathrm{I}-\mathrm{N}$} & \multicolumn{3}{|c|}{$\mathrm{N}-\mathrm{Sm} \mathrm{A}$} \\
\hline & $P_{\mathrm{I}-\mathrm{N}}^{*}$ & $\rho_{\mathrm{I}}^{*}$ & $\rho_{\mathrm{N}}^{*}$ & $P_{\mathrm{N}-\mathrm{Sm} A}^{*}$ & $\rho_{\mathrm{N}}^{*}$ & $\rho_{\mathrm{Sm} \mathrm{A}}^{*}$ \\
\hline $\begin{array}{c}\text { SWSC } \\
\text { (compression) }\end{array}$ & 1.425 & 0.0993 & 0.1016 & 1.675 & 0.1085 & 0.1132 \\
\hline $\begin{array}{c}\text { SWSC } \\
\text { (expansion) }\end{array}$ & 1.325 & 0.0968 & 0.0993 & 1.675 & 0.1099 & 0.1155 \\
\hline $\begin{array}{c}\text { SRS } \\
\text { (compression) }\end{array}$ & 1.225 & 0.1117 & 0.1157 & 1.825 & 0.145 & 0.147 \\
\hline $\begin{array}{c}\text { SRS } \\
\text { (expansion) }\end{array}$ & 1.225 & 0.1119 & 0.1157 & 1.775 & 0.1387 & 0.1431 \\
\hline $\mathrm{HSC}$ & 1.190 & 0.0914 & 0.0932 & 1.540 & 0.1061 & 0.1095 \\
\hline
\end{tabular}

A phase. The authors of Ref. 5 already recognized that the larger effective size of the SRS molecules, as a consequence of the longer range of the interaction in comparison to the HSC system (the SRS potential becomes zero at an intermolecular distance roughly $12 \%$ greater than the HSC potential), was responsible for the enhancement of excluded volume effects. We can extend this latter argumentation to reconcile the apparently opposite behavior of the SRS fluids with $\left(L^{*}=4\right.$ and $\left.T^{*}=1\right)$ and $\left(L^{*}=5\right.$ and $\left.T^{*}=5\right)$. The effective size of the SRS molecules is a dynamic concept, meaning that it depends on temperature. Figure 1 shows that system energies greater than unity $(U / \varepsilon>1)$ are related to reduced distances smaller than unity $\left(d_{m} / \sigma<1\right)$, i.e., effective molecular sizes smaller than in the HSC fluid. This high energy region of the potential energy is more extensively explored at high temperature and, in fact, in our simulations at $T^{*}=5$ we find that $U / \varepsilon>5$ for all the states relevant for the liquid crystalline phase transitions. In addition, the radial distribution $g(r)$ for this fluid at $T^{*}=5$ remains significant at $r / \sigma<1$ (see Fig. 5), in contrast to the results of Earl et al. at $T^{*}=1$ that yielded radial functions with $g(r / \sigma<1) \approx 0$ (Fig. 5 of Ref. 5). We conclude that the effective size of the molecules of the SRS fluid is greater at $T^{*}=1$ but smaller at $T^{*}=5$ in comparison to the HSC fluid, and we note that at some intermediate temperature, the SRS and HSC fluids of same elongation can be expected to display similar (at least to a certain extent) liquid crystalline phase diagrams. This latter consideration may be useful when considering the HSC fluid as a reference system in perturbative treatments of soft potentials (for instance of the SRS fluid).

\section{Residual pair and multiparticle entropy}

Figures 7 and 8 depict the evolution with increasing density of the excess entropy, $s_{\mathrm{ex}}$, the residual pair, $s_{2}$, and multiparticle, $\Delta s=s_{\mathrm{ex}}-s_{2}$, entropies, as well as of the translational and orientational components of the residual pair entropy, $s_{2}^{\mathrm{tr}}$ and $s_{2}^{\text {or }}$, obtained in our simulations of the SWSC

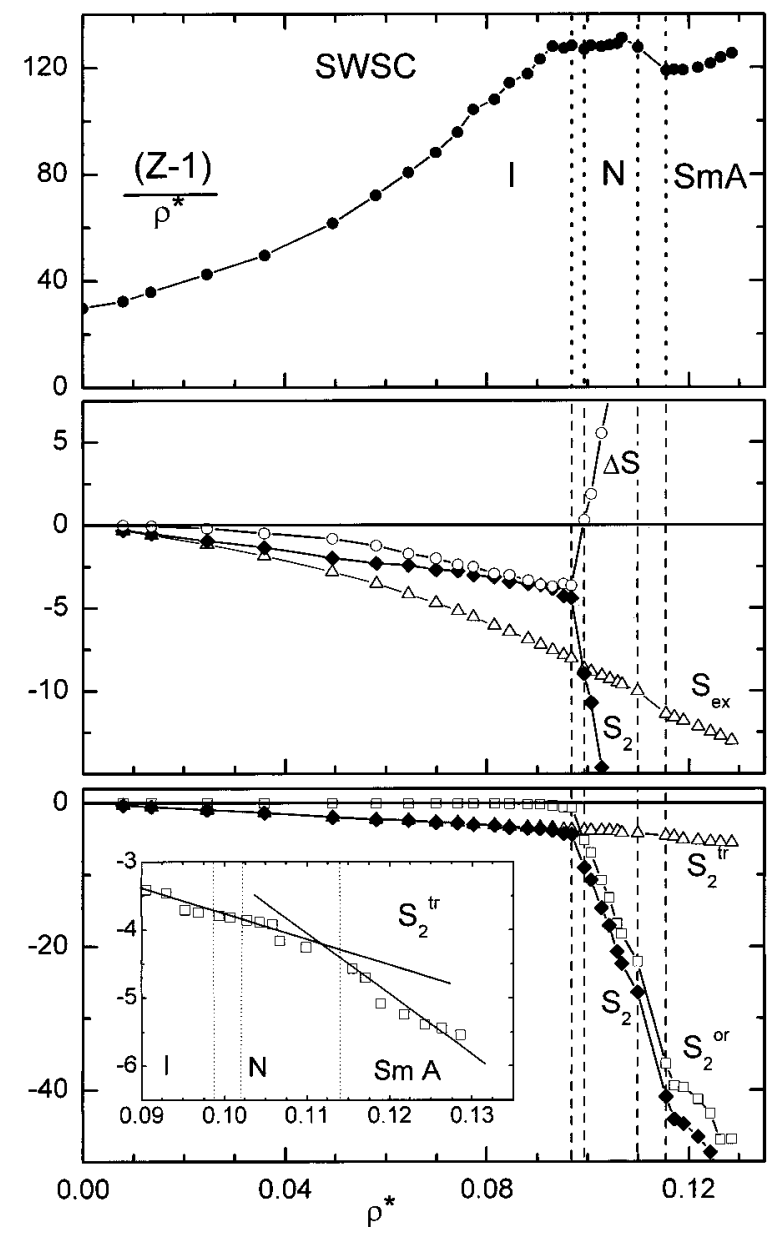

FIG. 7. Configurational entropies for the SWSC fluid vs the reduced number density $\rho^{*}=\rho / \sigma^{3}$. (Top panel) integrand of Eqs. (3) and (9), $(Z-1) / \rho^{*}(Z$, the compressibility factor). The value at $\rho^{*}=0$ is the reduced second virial coefficient of the model at $T^{*}=5$ (see Ref. 6 for its analytical expression). (Middle panel) excess entropy, $s_{\mathrm{ex}}$, pair entropy, $s_{2}$, and residual multiparticle entropy, $\Delta s=s_{\mathrm{ex}}-s_{2}$ [see Eqs. (3)-(9) for definitions]. (Bottom panel) decomposition of the pair entropy into translational and orientational contributions ( $s_{2}^{\mathrm{tr}}$ and $s_{2}^{\text {or }}$, respectively). The inset in the bottom panel illustrates an apparent change of slope of $s_{2}^{\text {tr }}$ after the nematic-smectic A transition (the straight lines are to guide the eye).

and SRS fluids. The calculations were performed employing Eqs. (3)-(9) and merging, for each model fluid, the simulations of the MC compression run up to $P^{*}=0.90$ with those of the expansion run for $P^{*} \geqslant 1.0$.

The behavior of the different entropies follow a similar qualitative trend for the SWSC and SRS fluids, which agrees with the results of Costa et al. for the HSC fluid. ${ }^{17}$ For both systems, $\Delta s$ is slightly negative at low densities and decreases slowly with growing density within the isotropic phase of the fluids. Not unexpectedly, this trend reverses at the isotropic-nematic transition where $\Delta s$ undergoes a sudden inflexion and grows rapidly toward high positive values. Such behavior constitutes the basis of the $\Delta s=0$ criterion proposed by Costa et al. to allocate the I-N phase boundary. In fact, the interpolation of the $\Delta s$ values obtained in our computations intersect the density axis at values of $\rho$ that lie between the I-N coexistence densities (taken to be the densities of the boundary isotropic and nematic sates in the MC simulations, see Table V). Namely, for the SWSC fluid (Fig. 


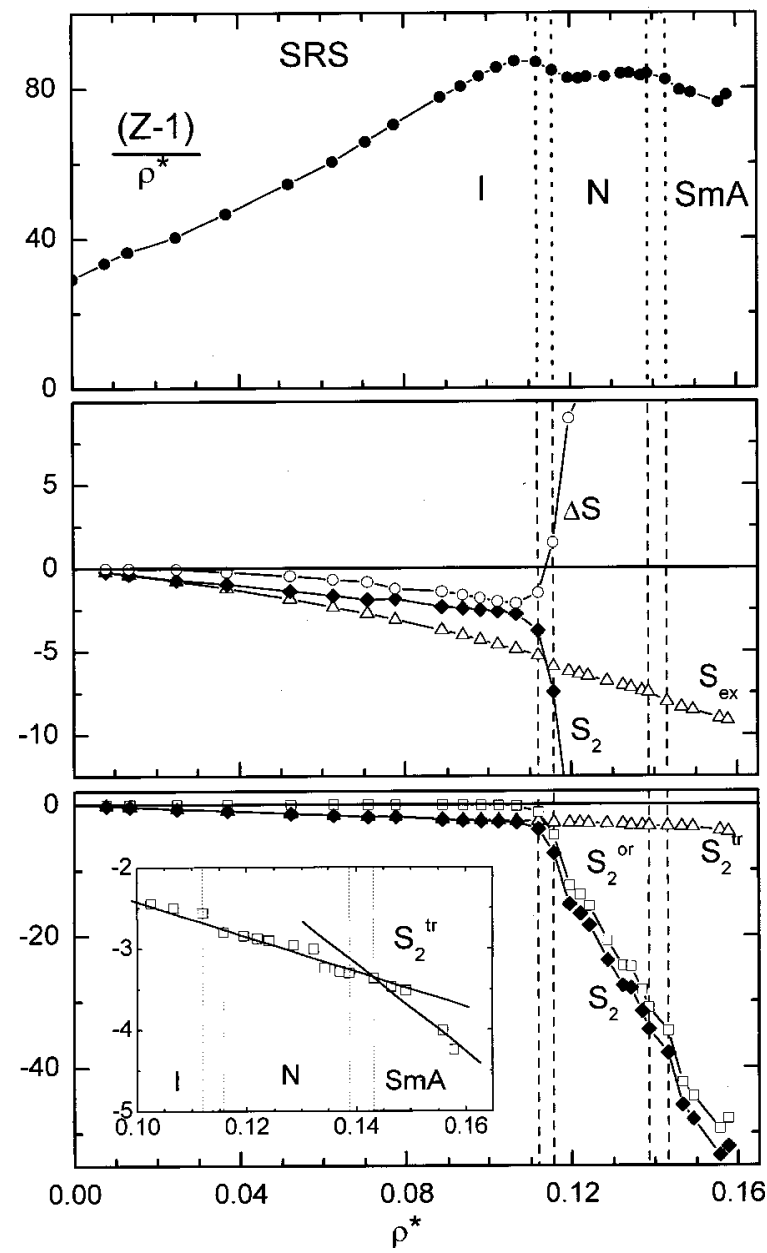

FIG. 8. Configurational entropies for the SRS fluid vs the reduced number density $\rho^{*}=\rho \cdot \sigma^{3}$. The same representations and notations are presented as in the three panels in Fig. 7. The value for $(Z-1) / \rho^{*}$ at $\rho^{*}=0$ in the top panel, which should correspond to the second virial coefficient of the model, is obtained by linear extrapolation of the first two values in the simulated curve.

7) the intersection takes place at $\rho=0.099$, and for the SRS fluid (Fig. 8) at $\rho=0.114$. In view of such good agreement, it can be expected that the $\Delta s=0$ criterion may safely be applied to liquid crystal models of linear molecules with more complex attractive or repulsive interactions. This result was not necessarily expected since, as already mentioned above, a similar entropy criterion was found not to be entirely successful when applied, for instance, to the freezing transition of atomic fluids with attractive interactions. ${ }^{15}$

It is important to note that, according to our simulations, the inflexion of $\Delta s$ toward positive values appears as a transitional effect. The behavior of $\Delta s$ all through the isotropic phase, including the isotropic state at the I-N coexistence, does not display any alteration, beside fluctuations, related to the incipient ordering transition to the nematic phase. It is only at the I-N coexistence states that the inflexion of $\Delta s$ takes place; at coexistence $\Delta s<0$ for the isotropic state and $\Delta s>0$ for the nematic state. This result, which we observe for both the SWSC and the SRS fluids, differs qualitatively from the interpretation of Costa et al. $^{17}$ of the change in the behavior of $\Delta s$ in the HSC system in terms of a pretransitional ordering in the fluid anticipating the macroscopic I-N transformation. However, according to a private communication by the authors, ${ }^{17}$ such interpretation was conditioned by the accidental overlooking of a significant scale factor. The revised analysis of the data of Costa et al., yields a packing fraction at which $\Delta s=0$ of $\eta \approx 0.40$ (i.e., a value essentially consistent with the location of the I-N transition of the HSC fluid, $\eta_{\mathrm{I}-\mathrm{N}}=0.407-0.415$, from MC simulations ${ }^{3}$ ), in comparison to the value of $\eta \approx 0.38$ (i.e., a pretransitional value) initially considered in Ref. 17. We therefore cannot confirm, for the I-N transition of the HSC, SWSC or SRS fluids, the appearance of any onset of the pretransitional ordering proposed in previous studies for the freezing, gas-liquid or mixing transitions of atomic fluids. ${ }^{14-16}$

A further inspection of Figs. 7 and 8, reveals that the sudden divergence of $\Delta s$ at the isotropic-nematic transition is linked to a similar behavior (although with reverse sign) for the residual pair entropy, $s_{2}$. On the other hand, the excess entropy, $s_{\mathrm{ex}}$, decreases at roughly constant rate within the whole range of densities investigated and remains apparently insensitive to the liquid crystal phase transitions undergone by the fluid (except for the weak discontinuity due to the entropy of transition). Furthermore, it is unequivocally observed that, for both the SWSC and SRS models, it is the orientational component of the pair entropy, $s_{2}^{\text {or }}$, which, as expected, accounts for the behavior of $\Delta s$ at the I-N transition. After the entrance of the fluid into the nematic phase, $s_{2}^{\text {or }}$, decreases with growing density at a rapid and roughly constant rate which is mantained even within the smectic phase in spite of the stabilization (within fluctuations) of the order parameter at values asymptotically close to unity (Figs. 2 and 4). On the other hand, the evolution of the translational pair entropy, $s_{2}^{\text {tra }}$ [Eq. (4)], which does not respond appreciably to the isotropic-nematic transition, does seem to show sensitivity to the translational ordering imposed by the smectic A phase. Indeed, the first derivative of $s_{2}^{\text {tra }}$ apparently changes abruptly at the nematic-smectic A transition. This is illustrated in the bottom panels of Fig. 7 (SWSC fluid) and of Fig. 8 (SRS fluid) in which the mean slopes followed by the $s_{2}^{\text {tra }}$ values before and after the $\mathrm{N}-\mathrm{Sm} \mathrm{A}$ transition are indicated roughly by straight lines. However, we have to remark that our results regarding the behavior of $s_{2}^{\text {tra }}$ within the smectic phase cannot be conclusive. This is due to the limited size of the simulation box employed in our study, which has prevented the full convergence of the pair correlation function to its asymptotic bulk value $[g(r) \rightarrow 1$ as $r \rightarrow \infty]$ and, thus, the correct computation of the radial integral in Eq. (4) for $s_{2}^{\text {tra }}$. Hence, a definite assessment of the use of $s_{2}^{\text {tra }}$ to characterize liquid crystal transitions in combination with $\Delta s$ and $s_{2}^{\text {or }}$ requires further simulation studies with a larger system size.

\section{SUMMARY AND CONCLUSIONS}

The isothermal-isobaric Monte Carlo study reported in this work for fluids of (a) hard spherocylinders with an attractive square-well (SWSC) and (b) repulsive soft spherocylinders (SRS), in both cases for a length-to-breadth ratio $L^{*}=5$ and at a reduced temperature $T^{*}=5$, has provided evidence for differentiated liquid crystal phases (namely, 
nematic and smectic A phases) in these two model fluids at reduced pressures ranging $P^{*}=0.1-2.2$. The main object of such study has been the investigation of the effect on the phase diagram of the introduction of either an attractive well or a soft repulsive core with respect to the better known hard spherocylinder fluid (HSC).

We find that the introduction of the attractive square well in the SWSC fluid induces a moderate but appreciable shift of the isotropic-nematic and nematic-smectic phase transitions toward higher packing fractions with respect to the HSC fluid. Thus, the presence of the attractive well imposes configurational constrains that oppose the entropic (excluded volume) contribution to the free energy, with the effect of a stabilization of the isotropic phase with respect to the nematic phase, and also this latter one with respect to the smectic phase.

The substitution of the hard core by a soft repulsive core leads to a much more significant shift of both the I-N and the N-Sm A transitions (especially of the latter one) toward higher densities. This effect can be attributed to the smaller magnitude in the SRS fluid of entropic excluded volume effects in comparison to its hard core counterparts. The soft core interaction allows for a greater packing of the fluid before the entropic contribution to the free energy forces the liquid crystalline transition. The apparent discrepancy of this result with an earlier study of the SRS fluid with $L^{*}=4$ and $T^{*}=1$ (Ref. 5) (which yielded I-N and N-Sm A transitions at smaller pressures and densities than the HSC fluid) can be reconciled by recognizing that the effective size of the molecules of the SRS fluid is larger at $T^{*}=1$ but smaller at $T^{*}=5$ in comparison to the HSC fluid. We conclude that at some intermediate temperature, the SRS and HSC fluids of same elongation can be expected to display similar liquid crystalline behaviors which may be useful when considering the HSC fluid as a reference system in perturbative treatments of soft potentials.

A brief reference to the extensively studied Gay-Berne (GB) fluid $^{2,20}$ and its hard repulsive core counterpart, the hard ellipsoid (HE) fluid, seems in order within this context. The isotropic-nematic transition for the HE fluid with axis ratios $\kappa=3$ and 4 is estimated to take place at reduced densities $\rho^{*}=0.325$ and 0.210 , respectively. ${ }^{21}$ The comparison of these values with those of the GB fluid is not straightforward, due to the three additional parameters (plus temperature) that characterize the pair interaction in this latter model. We will refer to the recent simulations ${ }^{22}$ for GB fluids employing a set of typical parameters $\left(\mu=2, \nu=1, \kappa^{\prime}=5\right.$, in the usual notation) and temperatures $T^{*} \leqslant 2$. Under these conditions the isotropic-nematic transition is found at significantly smaller densities with respect to the HE fluid, namely, $\rho^{*}=0.305$ and 0.190 for molecular elongations $\kappa$ $=3$ and 4 , respectively. Whereas this trend is similar to the one found when confronting the SRS and HSC fluids at $T^{*}$ $=1$, from the results of Ref. 22 it is not clear how quickly the I-N coexistence densities of the GB fluid would shift to values larger than those of the HE fluid for temperatures $T^{*}>2$. In fact, the characteristic anisotropy of the attractive interaction in the GB model, favoring side-to-side orientation between neighboring molecules, is known to have a qualita- tive effect upon the phase diagram of the fluid, inducing the appearance of smectic A and B phases, which are absent in the HE fluid. Such large effects obscure to a large extent a comparison between these two latter fluids on the simpler basis of soft vs hard interactions employed in the present work.

Finally, we have tested and extended an entropy criterion to allocate liquid crystalline phase boundaries, recently proposed for the isotropic-nematic transition of the HSC fluid. ${ }^{17}$ In particular, the use of the zero of the residual multiparticle entropy $(\Delta s=0)$ to characterize the transition from the isotropic to the nematic phase performs well for the SWSC and the SRS fluids which indicates that such criterion may be safely be applied to liquid crystal models of linear molecules with more complex attractive or repulsive interactions. However, for the fluid models studied in this work the sudden change in $\Delta s$ takes place between the isotropic and the nematic states at coexistence and, hence, appears as a transitional effect without any onset (beyond fluctuations) of the pretransitional ordering proposed in previous studies for the freezing, gas-liquid or mixing transitions of atomic fluids.

With regard to the behavior of the pair and multiparticle contributions to the excess entropy at the N-Sm A transition, our study is inconclusive because of the limited size of the simulation box. Our results suggest an appreciable jump in the translational part of the pair entropy, $s_{2}^{\text {tra }}$, and in its rate of change, that we expect to be confirmed by extended simulations with a greater number of particles.

\section{ACKNOWLEDGMENTS}

This work has been supported by the Spanish Dirección General de Investigación Científica y Técnica under Grant No. PB98-0326 and Instituto de Salud Carlos III under Grant No. 01/1664. We also acknowledge support from the Plan Andaluz de Investigación (groups FQM-205 and FQM-319).

${ }^{1}$ M. P. Allen, G. T. Evans, D. Frenkel, and B. M. Mulder, Adv. Chem. Phys. 86, 1 (1993)

${ }^{2}$ L. F. Rull, Physica A 220, 113 (1995).

${ }^{3}$ S. C. McGrother, D. C. Williamson, and G. Jackson, J. Chem. Phys. 104, 6755 (1996).

${ }^{4}$ J. M. Ilnytskyi and M. R. Wilson, J. Mol. Liq. 92, 21 (2001).

${ }^{5}$ D. J. Earl, J. Ilnytskyi, and M. Wilson, Mol. Phys. 99, 1719 (2001).

${ }^{6}$ D. C. Williamson and F. del Río, J. Chem. Phys. 109, 4675 (1998).

${ }^{7}$ D. C. Williamson and Y. Guevara, J. Phys. Chem. 103, 7522 (1999).

${ }^{8}$ B. Martínez-Haya, L. F. Rull, A. Cuetos, and S. Lago, Mol. Phys. 99, 509 (2001).

9 (a) C. Vega and S. Lago, Mol. Phys. 72, 215 (1991); (b) P. Sevilla, S. Lago, C. Vega, and P. Padilla, Phys. Chem. Liq. 23, 1 (1991).

${ }^{10}$ K. M. Aoki and T. Akiyama, Mol. Cryst. Liq. Cryst. 299, 45 (1997).

${ }^{11}$ M. S. Al-Barwani and M. P. Allen, Phys. Rev. E 62, 6706 (2000).

${ }^{12}$ (a) H. S. Green, The Molecular Theory of Fluids (North-Holland, Amsterdam, 1952); (b) R. E. Nettleton and M. S. Green, J. Chem. Phys. 29, 1365 (1958); (c) D. C. Wallace, Phys. Rev. A 39, 4843 (1989); (d) A. Baranyai and D. J. Evans, ibid. 40, 3817 (1989).

${ }^{13}$ (a) T. Lazaridis and M. E. Paulaitis, J. Phys. Chem. 96, 3847 (1992); (b) T. Lazaridis and M. Karplus, J. Chem. Phys. 105, 4294 (1996).

${ }^{14}$ (a) P. V. Giaquinta and G. Giunta, Physica A 187, 145 (1992); (b) P. V. Giaquinta, G. Guinta, and S. Prestipino, Phys. Rev. A 45, R6966 (1992); (c) C. Caccamo, P. V. Giaquinta, and G. Guinta, J. Phys.: Condens. Matter 5,75 (1993); (d) P. V. Giaquinta, G. Guinta, and G. Malescio, Physica A 250, 91 (1998).

${ }^{15}$ E. Lomba, J. L. López-Martín, H. M. Cataldo, and C. F. Tejero, Phys. Rev. E 49, 5164 (1994). 
${ }^{16}$ F. Saija, S. Pretipino, and P. V. Giaquinta, J. Chem. Phys. 113, 2806 (2000), and references cited therein.

${ }^{17}$ D. Costa, F. Saija, and P. V. Giaquinta, Chem. Phys. Lett. 299, 252 (1998). According to a private communication by one of the authors (P.V.G.), a scale factor of roughly one order of magnitude was overlooked in the vertical scale of Figs. 2 and 3 of this reference. The correction for this factor brings the results to a better agreement with the location of the isotropic-nematic transition of the HSC fluid from simulation studies, and also with the magnitude of the values for the residual multiparticle entropy obtained in our present study of the SWSC and SRS fluids.

${ }^{18}$ M. P. Allen and D. J. Tildeley, Computer Simulation of Liquids (Oxford University Press, New York, 1999).
${ }^{19}$ F. del Río, E. Ávalos, R. Espíndola, L. F. Rull, G. Jackson, and S. Lago, Mol. Phys. (to be published).

${ }^{20}$ M. A. Bates and G. R. Luckhurst, J. Chem. Phys. 110, 7087 (1999), and references therein.

${ }^{21}$ (a) D. Frenkel and B. M. Mulder, Mol. Phys. 55, 1171 (1985); (b) P. J. Camp, C. P. Mason, M. P. Allen, A. A. Khare, and D. A. Kofke, J. Chem. Phys. 105, 2837 (1996). We consider the interpolation of the results of both of these works for the estimation of the I-N transition of the HE fluid with $\kappa=4$.

${ }^{22}$ J. T. Brown, M. P. Allen, E. Martín del Río, and E. De Miguel, Phys. Rev. E 57, 6685 (1998). 